\title{
Fine-tuning the catalytic activity by applying nitrogen-doped carbon nanotubes as catalyst supports for the hydrogenation of olefins
}

\author{
Emőke Sikora ${ }^{1}$ - Adrienn Kiss ${ }^{1}$ • Zsuzsa H. Göndör ${ }^{1}$ • Péter Pekker ${ }^{2}$. \\ Ferenc Kristály ${ }^{3}$ - Milán Szőrii ${ }^{1}$ Anita Rágyanszki ${ }^{4}$ • Béla Viskolcz ${ }^{1}$ • Béla Fiser ${ }^{1,5}$. \\ László Vanyorek ${ }^{1}$ (D)
}

Received: 28 October 2019 / Accepted: 9 December 2019 / Published online: 14 December 2019

(c) The Author(s) 2019

\begin{abstract}
Nitrogen-free multi-wall carbon nanotubes (MWCNTs) and N-doped bamboo-like carbon nanotubes (BCNTs) were synthesized by using catalytic vapor deposition (CVD) and used as catalyst support materials. Pd, Rh, Ru, and Ir have been deposited onto the nanotubes to achieve metal/nanotube catalysts. The catalytic activity of the samples was fine-tuned by changing the type of support. BCNT supported $\mathrm{Pd}$ and $\mathrm{Rh}(\mathrm{Pd} / \mathrm{BCNT}, \mathrm{Rh} / \mathrm{MWCNT})$ catalysts were found to be the most active for liquid phase hydrogenation of octadecene amongst these samples. The initial olefin hydrogenation rate of the Pd/BCNT sample was slightly higher than the corresponding MWCNT-supported catalyst. Based on the hydrogenation reaction, the performance of these catalyst had been ranked as follows: $\mathrm{Pd} / \mathrm{BCNT} \approx \mathrm{Rh} / \mathrm{MWCNT}>\mathrm{Pd} /$ $\mathrm{MWCNT}>\mathrm{Rh} / \mathrm{BCNT}>>\mathrm{Ir} / \mathrm{MWCNT}>\mathrm{Ru} / \mathrm{BCNT}>\mathrm{Ir} / \mathrm{BCNT}>\mathrm{Ru} / \mathrm{MWCNT}$. The structural properties of chemisorbed Pd on MWCNT and N- BCNT were also characterized by means of computational chemical methods in order to shed some light on the nature of metal binding properties of $\mathrm{N}$-doped and undoped surfaces. The calculations shown preference towards the edges of the surfaces which is in good agreement with the experimental findings.
\end{abstract}

Electronic supplementary material The online version of this article (https://doi.org/10.1007/s1114 4-019-01705-7) contains supplementary material, which is available to authorized users.

László Vanyorek

kemvanyi@uni-miskolc.hu

1 Institute of Chemistry, University of Miskolc, Miskolc-Egyetemváros 3515, Hungary

2 Research Institute of Biomolecular and Chemical Engineering University of Pannonia, Veszprém 8200, Hungary

3 Institute of Mineralogy and Geology, University of Miskolc, Miskolc-Egyetemváros 3515, Hungary

4 Department of Chemistry, York University, 4700 Keele Street, Toronto, ON M3J 1P3, Canada

5 Ferenc Rákóczi II. Transcarpathian Hungarian Institute, Beregszász 90200, Ukraine 
Keywords Carbon nanotubes $\cdot$ Catalyst $\cdot$ BCNT $\cdot$ MWCNT $\cdot$ Hydrogenation

\section{Introduction}

The most widely-used industrial catalysts are nowadays activated carbon-based (AC) or zeolite-supported materials, with different noble metals [1-5]. These catalysts can ensure high product selectivity and efficient conversion, despite the slower reaction rate, which is due to diffusion limitation originated from their microporous structures $[6,7]$. Such reactant-product mass transport issue can be avoided by nanostructured carbon material support, like carbon nanotubes (CNT), which therefore accelerate the required reaction [8,9]. Furthermore, extraordinary thermal conductivity [10] and electronic properties [11] can also make these nanostructured carbon materials as excellent candidates for catalyst supporters. Due to curvature of the nanotube wall, the distorted electron configuration can provide interaction sites for catalytically active phase [6] such as rhodium, palladium, ruthenium, iridium particles in hydrogenation processes [8]. Multi- walled carbon nanotubes (MWCNTs) are used frequently in these catalytic applications due to their easier production on large scale [12]. For instance, MWCNT supported palladium nanoparticles ( $\mathrm{Pd} / \mathrm{MWCNT}$ ) is found efficient catalyst for hydrogenation of biomass-derived levulinic acid [13]. Due to their extended high-energy edges, bamboo-like carbon nanotubes (BCNTs) are more active CNTs as sorbents than the MWCNTs. There- fore, they are also preferred to use in heterogeneous catalysis as a support material [14].

The interaction strength between the catalyst support and the metallic phase can also be modified by electronic modification of the nanotubes by incorporation of heteroatoms such as nitrogen [15]. Nitrogen doping leads to the formation of defective sites in the carbon nanotube structure [16]. These defects also act as anchoring sites for well dispersed catalytically active metal nanoparticles making metallic catalyst supported on N-CNTs. There are many applications of such CNT-supported catalysts, for instance, in hydrogenation [17] and dehydrogenation processes [18], oxidation reactions [19], Fisher- Tropsch synthesis [20] and hydro-desulfurization [21].

To combine the above-mentioned features of the BCNTs, nitrogen doping and metal nanoparticles, selective hydrogenation of nitrobenzophenone to aminobenzophenone over Pd supported on BCNTs $(\mathrm{Pd} / \mathrm{N}-\mathrm{CNT})$ was investigated and $\mathrm{Pd} / \mathrm{N}$ CNTs exhibited a higher selectivity towards aminobenzophenone than $\mathrm{Pd} / \mathrm{CNT}$ and $\mathrm{Pd}$ on activated carbon $(\mathrm{Pd} / \mathrm{AC})$ [22]. Furthermore, $\mathrm{Pd} / \mathrm{N}-\mathrm{CNT}$ are promising catalysts for the selective reduction of cinnamaldehyde [23]. The Pd/N-CNT catalyst also showed higher activity at $823 \mathrm{~K}$ than the non-doped $\mathrm{Pd} / \mathrm{CNT}$ one in dehydrogenation of $\mathrm{C}_{2} \mathrm{H}_{6}$ in our previous work [24]. Nitrogen-doped graphene and carbon nanotube composite catalysts were also examined in electro reduction process of oxygen [25]. Higher activity was observed in the oxygen reduction reaction by using the N-doped catalyst compared to the Pd/AC system. According to this study, the increased activity can be attributed to the larger amount of total nitrogen and pyridinic nitrogen groups [25]. 
In this study, we aim to prepare and characterize catalysts involving $\mathrm{Pd}, \mathrm{Rh}, \mathrm{Ru}$, and Ir containing N-doped bamboo-like carbon nanotubes (BCNTs), and nitrogenfree multi-wall carbon nanotubes (MWCNTs) and test their catalytic performances in several hydrogenation reactions.

\section{Experimental section}

\section{Materials}

BCNTs and MWCNTs were prepared by Catalytic Vapour Deposition (CVD) technique. During this process volatile carbon sources were decomposed at $650{ }^{\circ} \mathrm{C}$ in the presence of catalytically active metals [26]. N-butylamine (Charlau Chem.) and acetylene (purity 4.0, Messer) was used for the synthesis of the BCNTs and nitrogen-free MWCNTs, respectively. Nickel(II) nitrate hexahydrate $\left(\mathrm{Ni}\left(\mathrm{NO}_{3}\right)_{2} \times 6 \mathrm{H}_{2} \mathrm{O}\right.$, Merck Chem.), cobalt(II) nitrate hexahydrate $\left(\mathrm{Co}\left(\mathrm{NO}_{3}\right)_{2} \times 6 \mathrm{H}_{2} \mathrm{O}\right.$, Reanal), iron(III) nitrate nonahydrate $\left(\mathrm{Fe}\left(\mathrm{NO}_{3}\right)_{3} \times 9 \mathrm{H}_{2} \mathrm{O}\right.$, Sigma Aldrich), magnesium oxide (Reanal), and a Patosolv mixture of aliphatic alcohols (Molar Chem.) were used as precursors for catalyst preparation. The noble metal containing catalysts were made from anhydrous palladium(II) acetate $\left(\mathrm{Pd}(\mathrm{OAc})_{2}\right.$, Fluorochem Ltd.), rhodium(II) acetate dimer ([Rh(OAc) $]_{2}$, Sigma Aldrich), ammonium hexachloroiridate(IV) $\left(\left(\mathrm{NH}_{4}\right)_{2} \mathrm{IrCl}_{6}\right.$, Sigma Aldrich) and ruthenium(III) chloride $\left(\mathrm{RuCl}_{3}\right.$, Sigma Aldrich). Nitrogen (purity 4.0, Merck) was used as inert atmosphere and carrier gas, while 1-decene, 1-tetradedecene and 1-octadecene (all from Sigma Aldrich) were hydrogenated by hydrogen (purity 4.0, Merck) in the presence of the prepared BCNT- and MWCNTsupported catalysts.

\section{Instrumentation}

To get information about the morphology the prepared catalysts and noble metal nanoparticles, images of samples were taken by using High Resolution Transmission Electron Microscopy (HRTEM, Technai G2, $200 \mathrm{kV}$ ). Samples used in HRTEM were prepared in a way that their aqueous suspension were dropped on 300 mesh copper grids (Ted Pella Inc.). The outermost diameters of the nanotubes were measured by analysing the HRTEM images using the ImageJ analysis software [27]. Purity of the CNTs was evaluated using a thermogravimetric analysis (TGA) applying a MOM Derivatograph-C device in oxidative atmosphere; the heating rate was $5{ }^{\circ} \mathrm{C} / \mathrm{min}$. The chemical state of the nitrogen in the graphitic lattice of BCNT was characterized by X-ray photoelectron spectroscopy (XPS) analysis using SPECS instrument with Phoibos 150 MCD 9 analyzer. The hydrogenation process was followed by Vertex 70 type Fourier Transformed Infrared Spectroscope (FTIR) in a gas cell with a $\mathrm{KBr}$ window. The presence and physiognomy of metal particles on the surface of the catalysts was investigated by X-ray diffraction (XRD), with a Bruker D8 Advance diffractometer ( $\mathrm{Cu}-\mathrm{K} \alpha$ source, $40 \mathrm{kV}$ and $40 \mathrm{~mA}$ generator settings), equipped with Vantec1 position sensitive detector (1 window opening). The parallel 
beam geometry of the instrument (by Göbel mirror) allowed measurements to be made at the milligram level, without the need of pressing powder specimens. Zero background sample holders ( $\mathrm{Si}$ crystal cut) eliminated the issue of sample transparency, no sample holder contribution presents. Inductively coupled plasma excitation optical emission spectrometry (ICP-OES, Varian) was applied to confirm the amount of metals in the catalysts.

\section{Synthesis of MWCNTs and BCNTs}

The CVD synthesis was carried out by applying a nitrogen atmosphere in a quartzreactor, which was placed in a tube furnace. The liquid carbon source, n-butylamine, was evaporated in a pre-furnace for the preparation of BCNTs, however this furnace was not used for MWCNT synthesis. The amine-containing carbon precursor evaporated immediately feeding the carbon source directly into the main furnace using a syringe pump and transported into the reactor space with a nitrogen carrier gas. The gas flow (nitrogen and acetylene), throughout the course of the MWCNT synthesis, was governed by flowmeters, and the ratio of the mixture was $\mathrm{C}_{2} \mathrm{H}_{2}: \mathrm{N}_{2} 1: 10$. The reaction took place on a catalyst bed which was placed in a quartz-bowl. The reaction was carried out on $5 \mathrm{wt} \% \mathrm{Ni} / \mathrm{MgO}$ catalyst in the case of BCNTs. The MWCNTs were produced with a cobalt- and iron-containing catalyst mixture $(2.5 \mathrm{wt} \%$ $\mathrm{Co}-2.5 \mathrm{wt} \% \mathrm{Fe} / \mathrm{MgO})$. The catalysts were prepared by a wet impregnation method, in a mixture of aliphatic alcohols. The latter was evaporated by rotary vacuum evaporator, and the products were dried further in a furnace to remove the medium from the catalyst. During the CVD synthesis, no reductive pre-treatment was used on the catalyst. After the CNT synthesis, the $\mathrm{Ni} / \mathrm{MgO}$ and $\mathrm{Co}-\mathrm{Fe} / \mathrm{MgO}$ catalysts were removed by boiling the samples in a solution of concentrated hydrochloric acid and ensuring continuous agitation and reflux for a $6 \mathrm{~h}$ long period. Finally, the CNTs was filtered off, washed with distilled water and dried at $105^{\circ} \mathrm{C}$ overnight.

\section{Decoration of MWCNTs and BCNTs with noble metal nanoparticles}

The noble-metal-decorated carbon nanotube supports were prepared by impregnation of nanotubes in an aqueous solution of metal salts. The carbon nanotubes were dispersed in $250 \mathrm{ml}$ of distilled water through ultrasonication. The solutions of metal precursors $\left(\mathrm{PdCl}_{2},\left[\mathrm{Rh}\left(\mathrm{CH}_{3} \mathrm{CO}_{2}\right)_{2}\right]_{2},\left(\mathrm{NH}_{4}\right)_{2} \mathrm{IrCl}_{6}\right.$ and $\mathrm{RuCl}_{3}$, all in $50 \mathrm{ml}$ dist. water $)$ were added to the CNT dispersions under ultra-sonication, and the suspensions were evaporated by rotary evaporator. The samples were dried and calcinated at $400{ }^{\circ} \mathrm{C}$ in a nitrogen atmosphere after being reduced at $400{ }^{\circ} \mathrm{C}$ in hydrogen flow. The catalytically active metal content of the prepared catalysts was $\sim 5 \mathrm{wt} \%$ and was confirmed by ICP measurements.

\section{Catalytic activity of MWCNTs and BCNTs supported catalysts}

Hydrogenation of 1-octadecene in octanol phase is chosen to compare the catalytic activities of the prepared catalysts, where the initial olefin concentration of 
$0.2625 \mathrm{~mol} / \mathrm{dm}^{3}$ was used. The hydrogenation reaction was conducted at $50{ }^{\circ} \mathrm{C}$ in three-neck round-bottom flask during continuous mixing at atmospheric pressure. The hydrogen flow-rate was $100 \mathrm{ml} / \mathrm{min}$. The reaction mixture contained $30 \mathrm{mg}$ of catalyst and olefin in an octanol solvent. The time history of the decaying double bond of olefin was followed by FTIR spectroscopy. Integration of the FTIR spectra over the range of $1610-1690 \mathrm{~cm}^{-1}$ was used to quantify the conversion of the hydrogenation reactions. This method was calibrated by using four different octadecene/octanol solution $\left(3 \times 10^{-1}-3 \times 10^{-2} \mathrm{~mol} / \mathrm{dm}^{3}\right.$, see SI Fig. 1). Three parallel measurements were conducted. In each case, the error rate $(\mathrm{SD} \%)$ of peak areas was found to be $1.2-1.9 \%$, which demonstrates the reliability of the measurements. Finally, the most active catalyst was tested in the hydrogenation of three different olefins (1-decene, 1-tetradecene, and 1-octadecene). In these reactions, the same reaction conditions were used as described above (30 mg catalyst, $50 \mathrm{ml}$ olefin/ octanol solution, $\mathrm{T}=50{ }^{\circ} \mathrm{C}, 100 \mathrm{ml} / \mathrm{min}, \mathrm{H}_{2}$ ).

\section{Computational methods}

To get more insight into the metal-nanotube interactions a $\mathrm{C}_{52} \mathrm{H}_{18}$ nanoflake was used as a model for the CNT surface in the calculations. The $\mathrm{N}$-doped versions (pyridinic and graphitic models) of the CNT surface models were built including a nitrogen into the original model system and removing the corresponding number of hydrogens and carbons.

A metal atom $(\mathrm{Pd})$ was placed close to the surface and the metal/surface interaction and the energetic properties of the complex formation were studied. The metal/ surface complexes were optimized using PBE0/6-31+G(d)/SDD level of theory as it is implemented into the Gaussian 09 program package [28]. All the structures presented in this work correspond to local minimum of the potential energy surface checked by normal mode analysis. Most of these complexes were found with PM6 as well. As found by Vazquez-Arenas et al. [29] and our calculations, structural and energetic parameters of graphene nanoflakes obtained from PM6 calculations are in very good agreement with those obtained at the PBE0/6-31+G(d) level of theory [30].

\section{Results}

\section{Characterization of the MWCNTs and BCNTs}

The purity of the MWCNT and the BCNT catalyst supports were evaluated through thermogravimetric analysis (TGA). The differential thermogravimetric curve (DTG) shows that the MWCNT sample began to oxidize at $\sim 430{ }^{\circ} \mathrm{C}$, while the initial decomposition temperature in the case of BCNT was $\sim 420{ }^{\circ} \mathrm{C}$ (SI Fig. 2a and b).

This low ignition temperature of CNT samples explains that the type of catalytic syn- thesis method, i.e. the residual catalyst metal particles and metal oxides, could promote the oxidation of carbon nanotubes, yielding a decrease 
in the theoretical ignition temperature. Furthermore, the defects on the mantle (curved surface) of the nanotubes could also contribute to a decrease in the ignition temperature. The measured carbon content of the MWCNT sample was 92.2 wt \%, and in the case of BCNT was $92.3 \mathrm{wt} \%$. The two types of carbon nanotube samples were also examined by electron microscopy. The split structure of the nitrogen-doped carbon nanotube is easily distinguishable from its non-doped counterparts (SI Fig. 2c, d).

The BCNT samples were tested by X-ray photoelectron spectroscopy (XPS) to study the incorporation mode of nitrogen atoms (SI Fig. 3). The deconvoluted N1s band in the XPS spectra can be used to determine the different binding types between $\mathrm{N}$ and $\mathrm{C}$ atoms. The incorporation of nitrogen atoms occurs similarly in the pyridine structure, as detected by the appearance of the peak at binding energy range of 398.3-399.8 eV in SI Fig. 3. The peak in the 401.0-401.4 eV interval proves the presence of graphitic nitrogen in the sample. These types are two common nitrogen incorporation modes for N-CNT [31]. Additional peak located at 404.0-405.6 eV binding energy can be assigned to either oxidized nitrogen species or molecular nitrogen stuck in the BCNT structure [29].

\section{Characterization of the MWCNT and BCNT supported catalyst}

The morphology of the catalytically active metal nanoparticles was examined by HRTEM on the different nanotube supported catalysts (SI Fig. 4).

The HRTEM image analysis revealed the size distribution of metal particles on the CNT surface. The average diameter of noble metal nanoparticles on the surface of BCNT was significantly higher than the observed MWCNT-supported composites. The average Ru nanoparticle diameter on the nitrogen-free MWCNT substrate was $2.9 \mathrm{~nm}$, while the $\mathrm{N}$ - BCNT-supported catalyst had about factor of three larger metal particles chemisorbed $(\mathrm{d}=8.6 \mathrm{~nm})$. The average Pd nanoparticle diameter on the surface of BCNT was significantly higher than the observed MWCNT-supported composites. The average $\mathrm{Ru}$ nanoparticle diameter on the nitrogen-free MWCNT substrate was $2.9 \mathrm{~nm}$, while the BCNT-supported catalyst had about factor of 3 larger metal particles chemisorbed $(\mathrm{d}=8.6 \mathrm{~nm})$. The average Pd nanoparticle diameter on the MWCNT support was $8.3 \mathrm{~nm}$, which was slightly smaller than that of the BCNT-supported sample $(14.9 \mathrm{~nm})$. The average particle size of Ir/MWCNT catalyst was $2.8 \mathrm{~nm}$, significantly smaller than the Ir/BCNT-supported sample $(7.1 \mathrm{~nm})$. Finally, the average $\mathrm{Rh}$ particle diameter for Rh/MWCNT catalyst $(3.8 \mathrm{~nm})$ is roughly the half of the $\mathrm{Rh} / \mathrm{BCNT}$ nano-composite (7.3).

$\mathrm{X}$-ray diffraction was used to confirm the presence of the elemental noble metal phases in the catalyst samples (SI Fig. 5). The broad peak of $2 \Theta$ at $26.5^{\circ}$ can be attributed to the $\mathrm{C}(002)$ reflection of the hexagonal graphite in both types of carbon nanotubes. The peaks of $2 \Theta$ at $40.5^{\circ}, 44.5^{\circ}$, and $47.5^{\circ}$ can be identified as (111), (101), (200) reflections in the iridium-, rhodium- and palladium-containing carbon nanotube supported catalysts, respectively. The reflection at $38.2^{\circ}$ belongs to $\mathrm{Ru}(100)$. 


\section{Catalytic activity tests of nanotube supported catalysts}

The catalytic activity of Ir-, Rh-, Ru-, and Pd-containing nanotube-supported samples were examined during the hydrogenation processes in liquid phase reactions, namely conversion of 1-octadecene to octadecane. The instantaneous octadecene conversion was monitored by FTIR intensity change of the $v(C=C)$ vibration band. As can be seen in Fig. 1, the most active catalysts among the MWCNT-supported samples were the rhodium- and the palladium-containing catalysts. Pure, unloaded nanotubes were also tested in catalytic hydrogenation, but the samples had no catalytic activity (SI Fig. 6).

The maximum conversion of octadecene was $96.5 \%$ on Rh/MWCNT after $80 \mathrm{~min}$, and $95.5 \%$ after two hours in the case of Pd/MWCNT. The same conversion using $\mathrm{Pd} / \mathrm{BCNT}$ was $94.4 \%$ after $80 \mathrm{~min}$ of hydrogenation, while this value in $\mathrm{Rh} / \mathrm{BCNT}$ was $95 \%$, after two hours. These results show that the palladiumand rhodium-supported catalysts are the most active, independent of the type of carbon nanotube support. The least active catalyst was the 5\% Ru/MWCNT catalyst. Catalytic performance of Ru/BCNT was higher (conversion of $40 \%$ after two hour) than its MWCNT-supported counterpart.

The hydrogenation reaction at Ir/BCNT was slightly slower compared to Ir/ MWCNT. The reason for this variation in catalytic performance can be explained by the absence of the incorporated nitrogen.

The high active metal catalyst in this study was palladium, on both types of carbon nanotube catalyst supports. Therefore, this was tested in the hydrogenation reaction of three 1 alkenes, namely 1-decene, 1-tetradecane and 1-octadecene. The reaction conditions for these hydrogenation processes were chosen

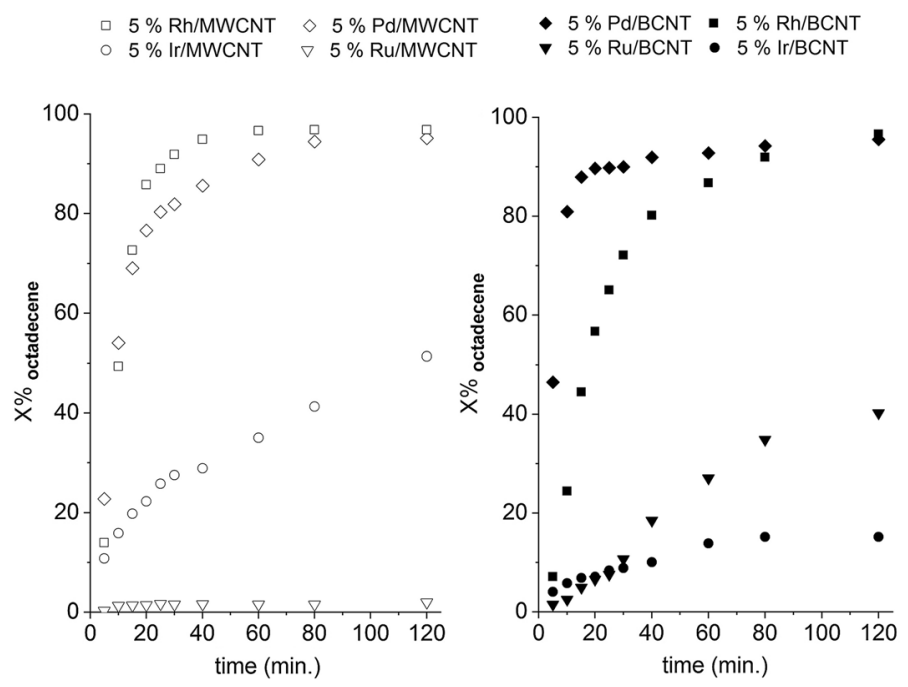

Fig. 1 Octadecene conversion vs. time of hydrogenation at $50{ }^{\circ} \mathrm{C}$, atmospheric pressure with used of MWCNT and BCNT supported Rh, Pd, Ir and Ru catalysts 
to be the same as in the previously described reactions. The time histories of the conversion are illustrated in Fig. 2.

The reaction rates of hydrogenation in all three olefins were similar when the same catalyst was used. An accelerated reaction rate was observed using $\mathrm{Pd} / \mathrm{BCNT}$ catalyst compared to the MWCNT samples. For instance, on the Pd/BCNT catalyst, $89.5 \%$ decene conversion was reached after $10 \mathrm{~min}$, while in the case of $\mathrm{Pd} /$ MWCNT, this result was only $66.2 \%$. it is worthy to mention here that our PM6 calculations showed also higher binding free energy towards the nitrogen doped surface in the case of palladium. Similar observations were made during the reactions of the other two olefins. It can therefore be concluded that the nitrogen-doped CNT-containing sample was the more effective catalyst compared to the non-doped nanotubesupported Pd catalyst. The different hydrogenation rates can be rationalized by the electronic configuration of the incorporated nitrogen and binding strength of metals onto the surface which is investigated in the next section.

\section{Computational results}

The structural and energetic properties of chemisorbed palladium on MWCNT and $\mathrm{N}$-doped BCNTs were studied using computational chemical tools. Nanoflake-metal model systems were designed to reduce the computational cost but preserve the reliability of the calculations [32]. In this way, several Pd-nanoflake complexes were created and characterized to model the system with the best catalytic activity. As the presence of pyridinic and graphitic nitrogen can be seen on the XPS spectra, two model systems were designed to study the effect of nitrogen doping (Fig. 3b and c).

In general, the formed complexes can be divided into two groups, based on the position of the metal, "edge"- or "centre"-type. The calculations show that the edge of the structures has a higher probability of binding metals in each case and the presence or absence of nitrogen does not affect the preference in this sense. However, the $\mathrm{N}$-doped model compounds can establish stronger
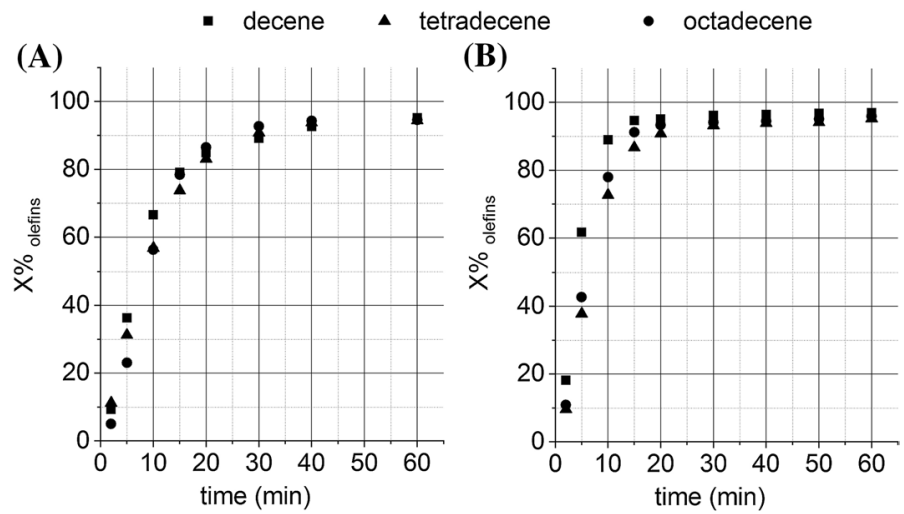

Fig. 2 Time evolution of the conversion (X\% $\%_{\text {olefins }}$ ) for hydrogenation of different 1-alkenes (decene, tetradecane, octadecene) at $50{ }^{\circ} \mathrm{C}$, atmospheric pressure using $\mathrm{Pd} / \mathrm{MWCNT}$ (a) and $\mathrm{Pd} / \mathrm{BCNT}$ (b) catalysts 
(A)
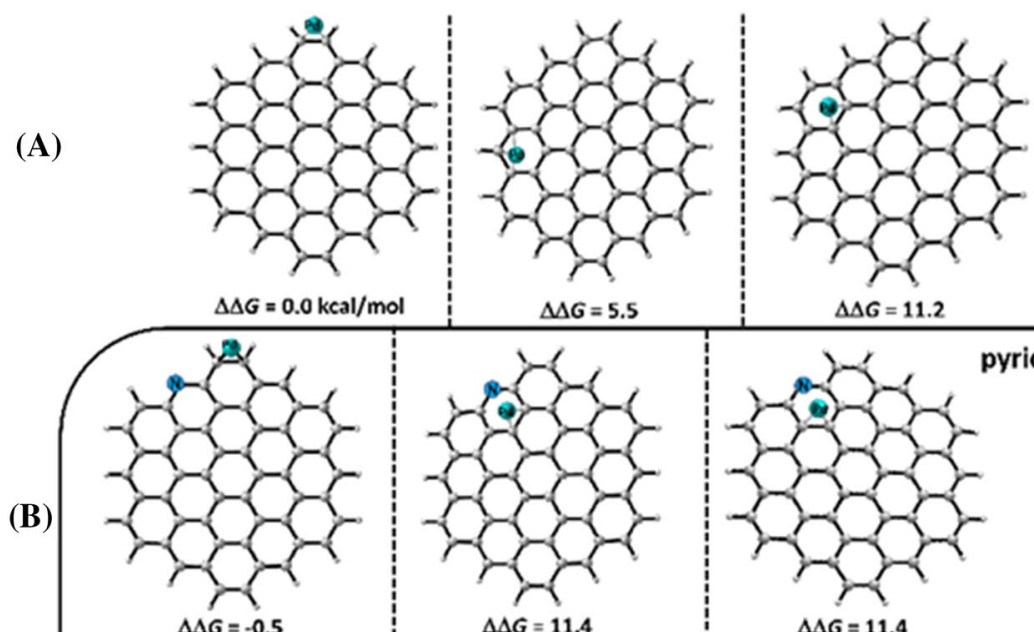

pyridinic
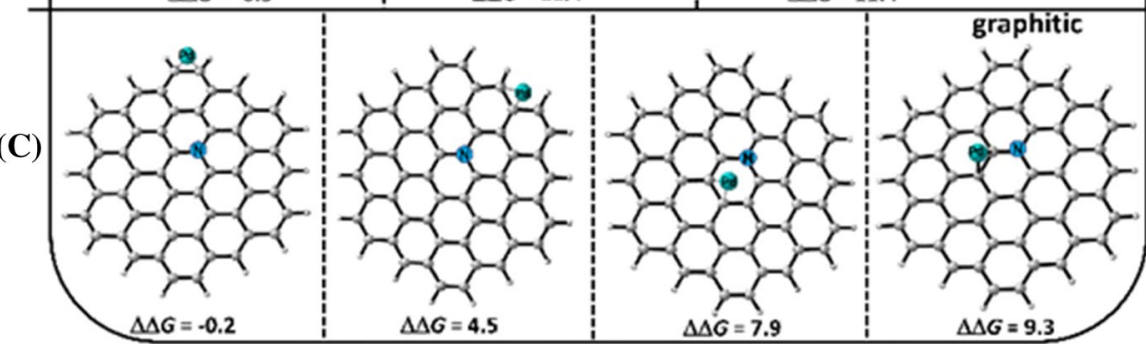

Fig. $3 \mathrm{Pd} /$ carbon nanotube $(\mathrm{Pd} / \mathrm{CNT})$ and $\mathrm{Pd} / \mathrm{N}$-doped bamboo-like carbon nanotube (Pd/N-CNT) model complexes (a and b, $\mathbf{c}$, respectively). The structures were optimized using PBE0/6-31+G(d)/SDD level of theory. The relative standard Gibbs energies $(\Delta \Delta G)$ are also shown in $\mathrm{kcal} / \mathrm{mol}$. The graphitic model system was positively charged, while the others were neutral

interaction with the Pd, than the nitrogen free CNT model measured by standard relative Gibbs free energy of metal binding $(\Delta \Delta G)$. But still the "edge" of the structures was preferred in the N-doped structures. The "edge"-type pyridinic and graphitic model complexes (Fig. 3, left, b and c) are the most favored ones $(\Delta \Delta G=-0.5$ and $-0.2 \mathrm{kcal} / \mathrm{mol}$, respectively). It should be noted that not the nitrogen, but the neighbouring carbon atoms or the carbons at the "edge" of the flakes will provide the best binding spots for the metal (Fig. 3b) which is in agreement with previous studies $[15,16]$ and experimental findings (see below).

The nitrogen incorporation into the system increased the binding potential of the "edge" of the structures. As can be seen on the HRTEM images (Fig. 4), the $\mathrm{Pd}$ particles mostly prefers to bind to the edges of the nanotubes. This is in good agreement with our computational findings, where the most stable structures show that the metal prefers to coordinate to the edge of the nanotube models (see above, Fig. 3). 

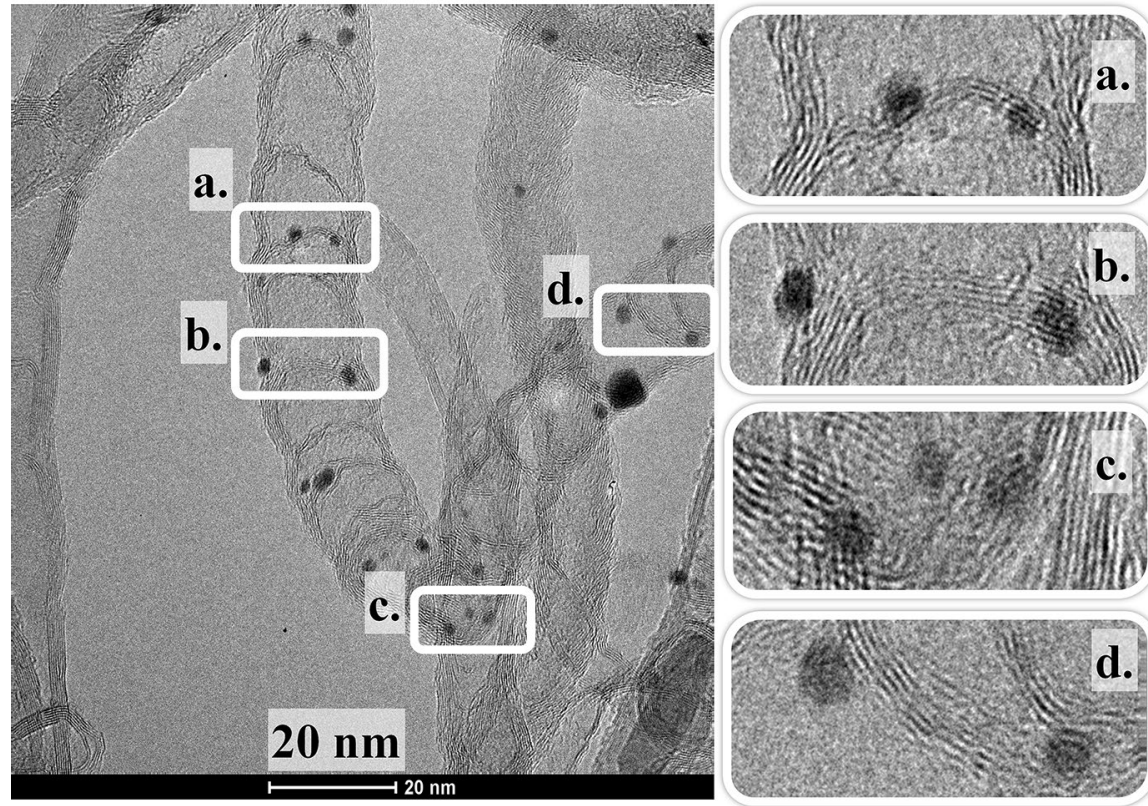

Fig. 4 HRTEM image about the location of Pd particles on the edges of N-doped bamboo-like carbon nanotube (BCNT) walls

\section{Conclusions}

Catalyst with $5 \mathrm{wt} \%$ metal (Pd, Rh, Ru, and Ir) content have been prepared. Nitrogen-free multi-wall carbon nanotubes (MWCNTs) and N-doped bamboo-like carbon nanotubes (BCNTs) were used as support materials and synthetized by means of catalytic vapour deposition method (CVD). The catalytic activity of the samples was fine-tuned by changing the type of support. The Ir-, Rh-, Pd-, and Rucontaining BCNT- and MWCNT-supported samples were compared through the hydrogenation reaction of 1-octadecene. The structural and energetic properties of Pd-containing MWCNT and BCNT catalyst models were also studied by means of calculations. The most active catalysts were the palladium- and rhodium-decorated nanotubes. The samples were ordered according to their catalytic performance: $\mathrm{Pd} /$ $\mathrm{BCNT}=\mathrm{Rh} / \mathrm{MWCNT}>\mathrm{Pd} / \mathrm{MWCNT}>\mathrm{Rh} / \mathrm{BCNT}>>\mathrm{Ir} / \mathrm{MWCNT}>\mathrm{Ru} / \mathrm{BCNT}>\mathrm{Ir} /$ BCNT $>$ Ru/MWCNT. The catalytic performance of Ru/MWCNT, Ir/MWCNT, Ru/ $\mathrm{BCNT}$, and Ir/BCNT catalysts was lower than expected. The best catalysts were the $\mathrm{Pd} / \mathrm{MWCNT}$ and $\mathrm{Pd} / \mathrm{BCNT}$, based on the comparison of three hydrogenation reactions of alkenes: 1-decene, 1-tetradecene and 1-octadecene. The catalytic activity of the $\mathrm{Pd} / \mathrm{BCNT}$ sample was higher than the $\mathrm{Pd} / \mathrm{MWCNT}$ sample, despite the greater palladium particle size. This enhanced activity can be interpreted as a result of the stronger complex formation in the case of the $\mathrm{Pd} / \mathrm{BCNT}$ systems which was shown by the calculations as well. All in all, palladium containing N-doped bamboo-like carbon nanotubes is the most promising catalyst within the studied set of samples. 
Acknowledgements Open access funding provided by University of Miskolc (ME). This research was supported by the European Union and the Hungarian State, co-financed by the European Regional Development Fund in the framework of the GINOP-2.3.4-15-2016-00004 project, aimed to promote the cooperation between the higher education and the industry. The GITDA (Governmental Information-Technology Development Agency, Hungary) is also gratefully acknowledged for allocating computing resources used in this work. The authors would like to thank Prof. Svend Knak Jensen (Aarhus University) for his help on various technical issues related to the calculations.

Open Access This article is licensed under a Creative Commons Attribution 4.0 International License, which permits use, sharing, adaptation, distribution and reproduction in any medium or format, as long as you give appropriate credit to the original author(s) and the source, provide a link to the Creative Commons licence, and indicate if changes were made. The images or other third party material in this article are included in the article's Creative Commons licence, unless indicated otherwise in a credit line to the material. If material is not included in the article's Creative Commons licence and your intended use is not permitted by statutory regulation or exceeds the permitted use, you will need to obtain permission directly from the copyright holder. To view a copy of this licence, visit http://creativecommons.org/licen ses/by/4.0/.

\section{References}

1. Upare DP, Park S, Kim MS et al (2017) Selective hydrocracking of pyrolysis fuel oil into benzene, toluene and xylene over CoMo/beta zeolite catalyst. J Ind Eng Chem 46:356-363. https://doi. org/10.1016/j.jiec.2016.11.004

2. Mendes PSF, Lapisardi G, Bouchy C et al (2015) Hydrogenating activity of Pt/zeolite catalysts focusing acid support and metal dispersion influence. Appl Catal A 504:17-28. https://doi. org/10.1016/j.apcata.2015.03.027

3. Gutiérrez A, Arandes JM, Castaño P et al (2011) Role of acidity in the deactivation and steady hydroconversion of light cycle oil on noble metal supported catalysts. Energy Fuels 25:3389-3399. https://doi.org/10.1021/ef200523g

4. Fu T, Li Z (2015) Review of recent development in co-based catalysts supported on carbon materials for Fischer-Tropsch synthesis. Chem. Eng. Sci. 135:3-20

5. Acres GJK, Bird AJ, Jenkins JW, King F (1981) The design and preparation of supported catalysts. In: Kemball C, Dowden DA (eds) Catalysis: Volume 4. The Royal Society of Chemistry, pp 1-30

6. Serp P, Corrias M, Kalck P (2003) Carbon nanotubes and nanofibers in catalysis. Appl Catal A 253:337-358

7. Pham-Huu C, Keller N, Ehret G et al (2001) Carbon nanofiber supported palladium catalyst for liquid-phase reactions an active and selective catalyst for hydrogenation of cinnamaldehyde into hydrocinnamaldehyde. J Mol Catal A 170:155-163. https://doi.org/10.1016/S1381-1169(01)00055 $-3$

8. Zhu J, Holmen A, Chen D (2013) Carbon nanomaterials in catalysis: proton affinity, chemical and electronic properties, and their catalytic consequences. ChemCatChem 5:378-401

9. Onoe T, Iwamoto S, Inoue M (2007) Synthesis and activity of the Pt catalyst supported on CNT. Catal Commun 8:701-706. https://doi.org/10.1016/j.catcom.2006.08.018

10. Balandin AA (2011) Thermal properties of graphene and nanostructured carbon materials. Nat Mater 10:569-581

11. Ouyang M, Huang J-L, Lieber CM (2002) Fundamental electronic properties and applications of single-walled carbon nanotubes. Acc Chem Res 35:1018-1025. https://doi.org/10.1021/ar0101685

12. Sairanen E, Karinen R, Borghei M et al (2012) Preparation methods for multi-walled carbon nanotube supported palladium catalysts. ChemCatChem 4:2055-2061. https://doi.org/10.1002/ cctc. 201200344

13. Yan K, Lafleur T, Liao J (2013) Facile synthesis of palladium nanoparticles supported on multiwalled carbon nanotube for efficient hydrogenation of biomass-derived levulinic acid. J Nanoparticle Res. https://doi.org/10.1007/s11051-013-1906-9

14. Liu K, Wang W, Xu Z et al (2009) Chirality-dependent transport properties of double-walled nanotubes measured in situ on their field-effect transistors. J Am Chem Soc 131:62-63. https://doi. org/10.1021/ja808593v 
15. Li YH, Hung TH, Chen CW (2009) A first-principles study of nitrogen- and boron-assisted platinum adsorption on carbon nanotubes. Carbon N Y 47:850-855. https://doi.org/10.1016/j.carbo n.2008.11.048

16. Zhou Z, Gao X, Yan J, Song D (2006) Doping effects of B and N on hydrogen adsorption in singlewalled carbon nanotubes through density functional calculations. Carbon N Y 44:939-947. https:// doi.org/10.1016/j.carbon.2005.10.016

17. Vanyorek L, Kristály F, Mihalkó A et al (2015) Synthesis and 1-butene hydrogenation activity of platinum decorated bamboo-shaped multiwall carbon nanotubes. React Kinet Mech Catal 116:371383. https://doi.org/10.1007/s11144-015-0906-4

18. Chen W, Ji J, Feng X et al (2014) Mechanistic insight into size-dependent activity and durability in Pt/CNT catalyzed hydrolytic dehydrogenation of ammonia borane. J Am Chem Soc 136:1673616739. https://doi.org/10.1021/ja509778y

19. Ovejero G, Sotelo JL, Rodríguez A et al (2007) Platinum catalyst on multiwalled carbon nanotubes for the catalytic wet air oxidation of phenol. Ind Eng Chem Res 46:6449-6455. https://doi. org/10.1021/ie070204p

20. Bahome MC, Jewell LL, Hildebrandt D et al (2005) Fischer-Tropsch synthesis over iron catalysts supported on carbon nanotubes. Appl Catal A 287:60-67. https://doi.org/10.1016/j.apcat a.2005.03.029

21. Yu Z, Fareid LE, Moljord K et al (2008) Hydrodesulfurization of thiophene on carbon nanofiber supported Co/Ni/Mo catalysts. Appl Catal B 84:482-489. https://doi.org/10.1016/j.apcat b.2008.05.013

22. Ombaka LM, Ndungu PG, Nyamori VO (2015) Pyrrolic nitrogen-doped carbon nanotubes: Physicochemical properties, interactions with Pd and their role in the selective hydrogenation of nitrobenzophenone. RSC Adv 5:109-122. https://doi.org/10.1039/c4ra12523a

23. Chizari K, Janowska I, Houllé M et al (2010) Tuning of nitrogen-doped carbon nanotubes as catalyst support for liquid-phase reaction. Appl Catal A 380:72-80. https://doi.org/10.1016/j.apcat a.2010.03.031

24. Vanyorek L, Halasi G, Pekker P et al (2016) Characterization and catalytic activity of different carbon supported pd nanocomposites. Catal Lett 146:2268-2277. https://doi.org/10.1007/s1056 2-016-1857-8

25. Ratso S, Kruusenberg I, Joost U et al (2016) Enhanced oxygen reduction reaction activity of nitrogen-doped graphene/multi-walled carbon nanotube catalysts in alkaline media. Int J Hydrog Energy 41:22510-22519. https://doi.org/10.1016/j.ijhydene.2016.02.021

26. José-Yacamán M, Miki-Yoshida M, Rendón L, Santiesteban JG (1993) Catalytic growth of carbon microtubules with fullerene structure. Appl Phys Lett 62:657-659. https://doi.org/10.1063/1.108857

27. Schneider CA, Rasband WS, Eliceiri KW (2012) NIH Image to ImageJ: 25 years of image analysis. Nat Methods 9:671-675

28. Frisch MJ, Trucks GW, Schlegel HB, et al (2009) Gaussian 09, Rev A.1. Gaussian, Inc.

29. Vazquez-Arenas J, Galano A, Lee DU et al (2016) Theoretical and experimental studies of highly active graphene nanosheets to determine catalytic nitrogen sites responsible for the oxygen reduction reaction in alkaline media. J Mater Chem A 4:976-990. https://doi.org/10.1039/C5TA06653K

30. Stewart JJP (2007) Optimization of parameters for semiempirical methods V: Modification of NDDO approximations and application to 70 elements. J Mol Model 13:1173-1213. https://doi. org/10.1007/s00894-007-0233-4

31. Matter PH, Zhang L, Ozkan US (2006) The role of nanostructure in nitrogen-containing carbon catalysts for the oxygen reduction reaction. J Catal 239:83-96. https://doi.org/10.1016/j. jcat.2006.01.022

32. Pykal M, Jurecka P, Karlicky F, Otyepka M (2015) Modelling of graphene functionalization. Phys Chem Chem Phys. https://doi.org/10.1039/C5CP03599F

Publisher's Note Springer Nature remains neutral with regard to jurisdictional claims in published maps and institutional affiliations. 